\title{
Dynamic collateral sensitivity profiles highlight challenges and opportunities for optimizing antibiotic sequences
}

\author{
Jeff Maltas ${ }^{1}$, Kevin B. Wood ${ }^{1,2, *}$ \\ 1 Department of Biophysics, University of Michigan, Ann Arbor, Michigan, United States of America \\ 2 Department of Physics, University of Michigan, Ann Arbor, Michigan, United States of America \\ *kbwood@umich.edu
}

\begin{abstract}
As traditional antimicrobial therapies fail at escalating rates, recent focus has shifted to evolution-based therapies to slow resistance. Collateral sensitivity-the increased susceptibility to one drug associated with evolved resistance to a different drug-offers a potentially exploitable evolutionary constraint, but the manner in which collateral effects emerge over time is not well understood. Here, we use laboratory evolution in the opportunistic pathogen E. faecalis to phenotypically characterize collateral profiles through evolutionary time. Specifically, we measure collateral profiles for 400 strain-antibiotic combinations over the course of 4 evolutionary time points as strains are selected in increasing concentrations of antibiotic. We find that collateral resistance dominates during early phases of adaptation, whereas a diverse set of collateral profiles are accessible with further selection. Using simple numerical simulations, we illustrate how these temporally dynamic profiles potentially impact sequential drug therapies. Finally, we show experimentally how dynamic collateral sensitivity relationships can create optimal dosing windows that depend on finely timed switching between drugs.
\end{abstract}

\section{Introduction}

Evolution of resistance continues to reduce the available set of drugs for successful treatment of bacterial infections, cancers and viral infections [1 6]. As traditional maximum tolerable dose treatments fail at climbing rates, evolution-based treatments have emerged as a promising method to prolong the efficacy of current drugs or even reverse resistance. These treatments include drug cycling [7 10], harnessing spatial dynamics [11 14, cooperation 15 18, adaptive therapy [19 21], and judicious use of drug combinations 22 29]. More recently there has been a growing focus on exploiting collateral sensitivity to slow or reverse evolution in bacteria and cancer 30 39].

Collateral evolution occurs when a population evolves resistance to a selecting drug and as a direct result exhibits increased or decreased resistance to a different drug.

While collateral sensitivity is a promising evolutionary therapy, a number of factors make its application to the clinic challenging; for example, collateral effects exhibit a high degree of heterogeneity 40,41], distinct collateral profiles arise from different selection pressures [42, collateral effects are often not repeatable [43], and many non-antibiotic environments can confer collateral sensitivity [44 49]. Despite these challenges, theoretical and laboratory studies have shown that control theoretic approaches may be used to counter, and even leverage, stochastic features of the evolutionary process to shape population outcomes [40,50]. Still, many fundamental questions about collateral sensitivity remain unanswered and are the focus of ongoing 
work. For example, the molecular mechanisms behind collateral sensitivity are known in relatively few cases 36,51, it is unclear the extent to which collateral profiles are conserved across diverse species 52], collateral drug pairs are difficult to identify in clinical settings [53, and somewhat surprisingly, little is known about how collateral profiles change under continued selection, with much of the work performed recently in cancer 37, 54.

In this work, we sought out to understand how collateral effects change over time in bacteria exposed to increasing antibiotic selection and, in turn, how these potentially dynamic collateral sensitivity profiles may influence the design of drug scheduling. Using laboratory evolution in Enterococcus faecalis, a gram-positive opportunistic bacterial pathogen typically found in the gastrointestinal tracts of humans [55 60], we measure collateral sensitivity and resistance profiles over time for 20 populations exposed to increasing concentrations of five drugs, yielding 400 strain-antibiotic susceptibility measurement combinations. These collateral profiles reveal a complex story. Collateral profiles are temporally dynamic and difficult to predict at a single-drug level. On the whole, however, we find that collateral resistance appears more frequently in early stages of adaptation, while further evolution leads to a diverse set of collateral outcomes. Using numerical simulations, we show that this early-stage collateral resistance manifests as a "tax" on drug switching when a new drug is introduced for the first time. As a result, short-term optimal treatments meant to minimize total resistance accumulation often preclude switching drugs. Finally, we show experimentally that optimal drug scheduling may require exploitation of transient time windows where collateral sensitivity is most likely to occur. Taken together, our results underscore the importance of measuring temporal collateral profile not only to better understand collateral evolution, but for any future work that hopes to harness collateral effects as a therapeutic option.

\section{Results}

\section{Collateral effects are temporally dynamic}

To investigate how collateral effects change over time in E. faecalis, we exposed four independent evolutionary replicates of strain V583 to escalating concentrations of a single drug over 8 days (approximately 60 generations) via serial-passage laboratory evolution (Fig 1, Materials and methods). In a previous study, we characterized collateral effects at the final endpoint of these evolutionary experiments [40]. In this work, we investigate the temporal progression of these collateral effects in a subset of five antibiotics with varying mechanisms of action (Table 1). To do so, we isolated a single colony from each population at 2-day intervals and measured dose-response curves to each of the antibiotics (Fig $1 \mathrm{~A})$.

Table 1. Table of antibiotics used in this study and their targets.

\begin{tabular}{|l|l|l|}
\hline Drug Name (Abbreviation) & Drug Class & Mechanism of Action \\
\hline Ceftriaxone (CRO) & $\beta$-Lactam & Cell wall synthesis inhibitor \\
\hline Ciprofloxacin (CIP) & Quinolone & DNA gyrase inhibitor \\
\hline Daptomycin (DAP) & Lipopeptide & Cell membrane insertion \\
\hline Doxycycline (DOX) & Tetracycline & 30S protein synthesis inhibitor \\
\hline Linezolid (LZD) & Oxazolidinone & 50S protein synthesis inhibitor \\
\hline
\end{tabular}

We quantified resistance and sensitivity by estimating the half-maximum inhibitory dose $\left(\mathrm{IC}_{50}\right)$ for each strain-antibiotic combination (Materials and Methods). In total, we estimated the $\mathrm{IC}_{50}$ for 400 strain-antibiotic combinations (20 evolving populations, measured against 5 antibiotics, at 4 evolutionary time points), each in (technical) 
replicates of three. For each measurement, we then calculated the collateral response $c \equiv \log _{2}\left(\mathrm{IC}_{50, \mathrm{Mut}} / \mathrm{IC}_{50, \mathrm{WT}}\right)$, the $\log _{2}$-scaled fold change in $\mathrm{IC}_{50}$ of the evolved strain relative to the ancestral V583 (Fig 1B). Resistance (direct or collateral) corresponds to $c>0$ while sensitivity corresponds to $c<0$ ( $c=0$ is indicated by the black dashed line). For a measurement to be deemed collaterally resistant or sensitive the measured mean value must be at least 3 times the standard error of the mean of the wild-type $\left(|c|>3 \sigma_{W T}\right)$.

Our results indicate that resistance to both the drug used for selection and the "unseen" testing drugs varies considerably over time. Surprisingly, these profiles are sometimes non-monotonic, with the same population exhibiting collateral resistance at one time point and collateral sensitivity at another (e.g. ceftrixaone (CRO)-selected strains tested against doxycycline (DOX), Linezolid (LZD)-selected strains tested against CRO). Additionally the variance in outcome is highly dependent on the testing drug. For example, evolved strains show a relatively narrow distribution of DOX across all 8 days of evolution, while resistance to $\mathrm{CRO}$ in the same strains is significantly more variable. We also observe varying levels of induced collateral sensitivity between tested drugs. For example, at no point do any of the 20 evolved strains become collaterally sensitive to LZD, and collateral sensitivity to DAP is rare at all evolutionary time points. This is particularly notable because LZD and DAP are frequently used as last line of defense antibiotics in the treatment of multidrug resistant gram-positive infections 61 64.

\section{Collateral resistance dominates early evolutionary outcomes}

To quantify how the propensity for collateral resistance changes over time, we next calculated the "instantaneous" collateral resistance (or sensitivity), which measures collateral effects at each time point relative to the previous time-point, rather than relative to the ancestral strain (Fig 2). For example, to investigate how the population changed between days 4 and 6 we calculate: $c_{\text {inst }} \equiv \log _{2}\left(\mathrm{IC}_{50, \mathrm{D} 6 \mathrm{Mut}} / \mathrm{IC}_{50, \mathrm{D} 4 \mathrm{Mut}}\right)$, where the $\mathrm{IC}_{50}$ 's are calculated at days 6 and 4 . This analysis reveals that the first two days of evolution are dominated by collateral resistance effects (91.25\%), while the subsequent 6 days of evolution confer collateral resistance at considerably reduced frequencies $(33.75 \%, 38.75 \%$, and $32.50 \%$ respectively). Further, after the first two days of evolution, the last 6 days also share a similar frequency of collateral sensitivity $(52.5 \%$, $55.0 \%$, and $57.5 \%$ respectively). One simple explanation of this phenomenon may be an abundance easily accessible, low-level resistance mutations, perhaps related to efflux pumps 65 67] or a general stress response 68, 69, that broadly confer low-level multidrug resistance. As the antibiotic concentration is increased the population is required to evolve more antibiotic-specific mutations that may be associated with collateral trade-offs. Future work will involve elucidating the mechanisms behind some of these differences and their genetic origins.

\section{Temporally dynamic collateral effects are difficult to predict from past measurements.}

While the distribution of collateral effects are similar for evolution between days 2 and 8 , collateral effects at the level of specific drugs are difficult to predict, even from collateral profiles measured at earlier times in the same strain. For example, one DAP-adapted population exhibits a significant increase in resistance to CRO between days 2 and 4, however days 4 to 6 and 6 to 8 both come with a significant collateral sensitivity. Similarly, the LZD-selected strains confer resistance to DAP on days 2 and 8 , but exhibit collateral sensitivity on days 4 and 6 . To quantify this effect, we calculated how often an observation from the current time step correctly predicted 


\section{A}

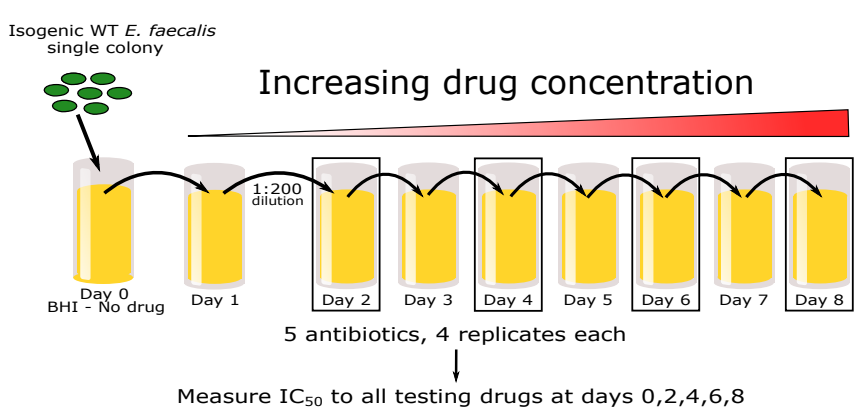

B
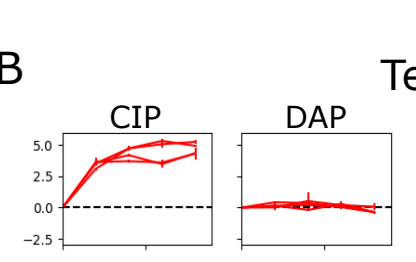

Testing Drug
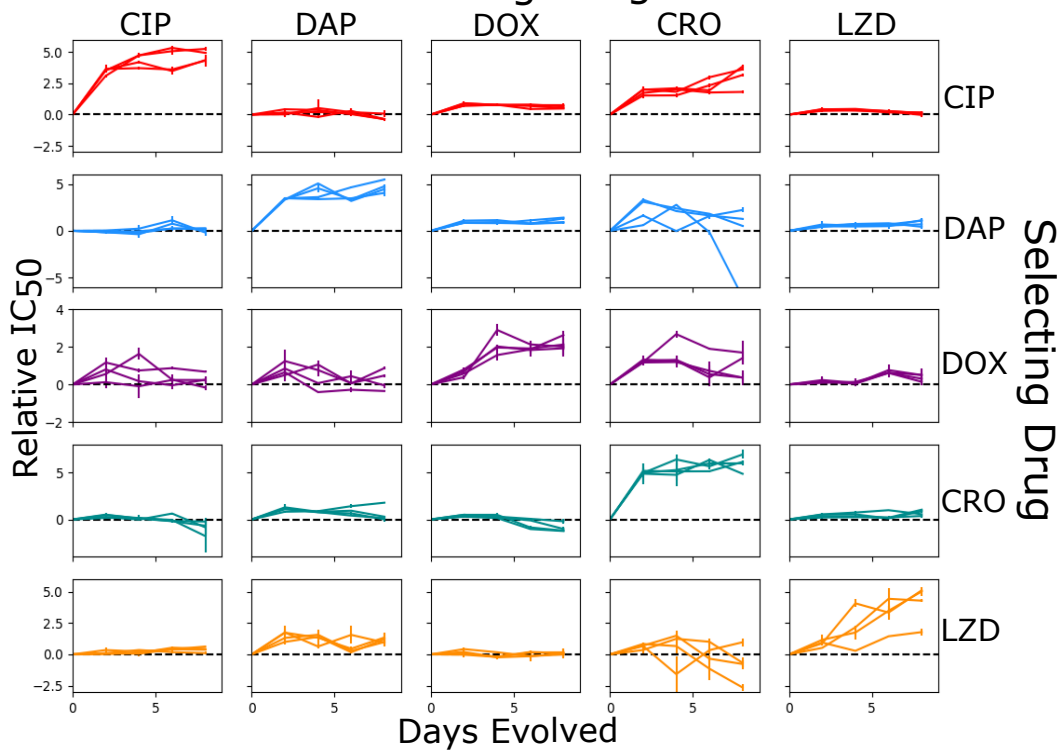

Fig 1. Laboratory evolution of E. faecalis reveals diverse, temporally dynamic collateral sensitivity profiles. (A) E. faecalis strain V583 was exposed to single antibiotics in escalating concentrations over the course of an 8-day serial-passage evolution experiment (roughly 60 total generations). Four independent populations were evolved in the presence of one of five selected antibiotics. The half-maximal inhibitory concentration $\left(I C_{50}\right)$ was estimated for a single isolate from each population at days 2, 4, 6 and 8. (B) Resistance/sensitivity measurements taken every two days for each of the 20 mutants to each of the 5 antibiotics quantified by the $\log _{2}$-transformed relative increase in the $I C_{50}$ of the testing drug relative to that of WT V583 cells (black dashed line at $y=0$ ). Rows represent the drug used to select the mutant (selecting drugs: Ciprofloxacin (CIP) = red, Daptomyacin $(\mathrm{DAP})=$ blue, Doxycycline $(\mathrm{DOX})=$ purple, Ceftrixone $(\mathrm{CRO})=$ teal, Linezolid $(\mathrm{LZD})$ = orange), whereas columns represent the drug used in the testing assay. Error bars represent the standard error of the mean (SEM) for each individual experiment. 
change in resistance measurement two days later. That is, if a particular isolate exhibited collateral sensitivity to a drug on day 2, how frequently did it also exhibit collateral sensitivity on day 4? Somewhat surprisingly, collateral profiles on day 2 correctly predict only $41 \%$ of day 4 collateral profiles. Similarly, day 4 only successfully predicted $37 \%$ of day 6 collateral profiles and day 6 accurately predicted $41 \%$ of day 8 profiles. These data indicate that, in contrast to resistance levels to the selecting drug, which tend to be non-decreasing over time, instantaneous collateral effects are largely uncorrelated after short periods (2 days; $10-20$ generations) of adaptation.

Despite the apparent unpredictability of the collateral profiles with continued selection, we asked whether antibiotics nevertheless shared some statistical similarities. To test this we calculated the Pearson correlation between each of the 5 testing conditions (Fig $\mathrm{S} 1 \mathrm{~A})$. We find that 5 of the 10 pairwise combinations produce a statistically significant correlation, all of which are negatively correlated. However, on a larger scale it appears none of the testing drugs produce profiles that are highly correlated or anti-correlated. This suggests that the diverse resistance mechanisms accrued over continued selection from mechanistically distinct antibiotics likely result in a lack of "global" collateral evolution rules between our tested antibiotics. Instead, profiles are not specific to a drug, but instead specific to the specific set of mutations acquired to that drug. It is possible that these correlations arise primarily due to highly nonlinear relationships between the variables, and therefore linear correlation measures are insufficient to quantify their relationships.

\section{Multi-drug sequences can minimize cumulative resistance to the applied drugs}

We also attempted to quantify how these temporally resolved collateral profiles might impact the effects of drugs used in different sequences (Fig 3). To do so, we consider a simple model where collateral effects accumulate linearly based on the resistance values measured for the selecting drug in question 40. Because collateral effects are measured on a $\log$ scale, this linear model reduces to one where fractional changes in resistance levels are multiplicative [70]. More specifically, in a system with $N$ drugs, the state at time $t$ is defined by an $N$-dimensional vector $S_{t}$, with each component of that vector the collateral value (i.e. log-scaled fold change in $\mathrm{IC}_{\text {text }} 50$ ) associated with a specific testing drug. The state of the system at the next time point, $t+1$, is then given by $S_{t+1}=S_{t}+\delta C$, where $\delta C$ accounts for the change in collateral profile due to selection in the currently applied drug. When an antibiotic is used for the first time, $\delta C$ is randomly drawn from one of the 4 (replicate) profiles measured on day 2 (i.e. one column of the matrix in Fig. 2B, upper left). However, for each subsequent use of that antibiotic, $\delta C$ is given by the collateral profile corresponding to the next temporal step on the chosen trajectory (i.e. by the same column but in the measured collateral matrix at the next time step). For example, the first time a population is exposed to CIP, $\delta C$ is drawn randomly from one of the 4 collateral profiles we observe on day 2 of CIP selection (for example, column 3 in Fig 2B, upper left matrix). However, when the population is exposed to CIP again, $\delta C$ will be given by the same column (column 3 ) of the collateral matrix measured on day 4 (Fig $2 \mathrm{~B}$, upper right). The goal of this model is to capture the measured temporal changes associated with repeated exposures to the same drug while neglecting higher-order temporal effects between different drugs applied multiple times (as measuring these higher-order effects would require an exponentially growing number of measurements because of the combinatorial explosion in possible trajectories). While this model is clearly an oversimplification of the full evolutionary dynamics, we have shown similar models can offer qualitatively accurate predictions in relatively short evolution experiments 40,44. And because of the 

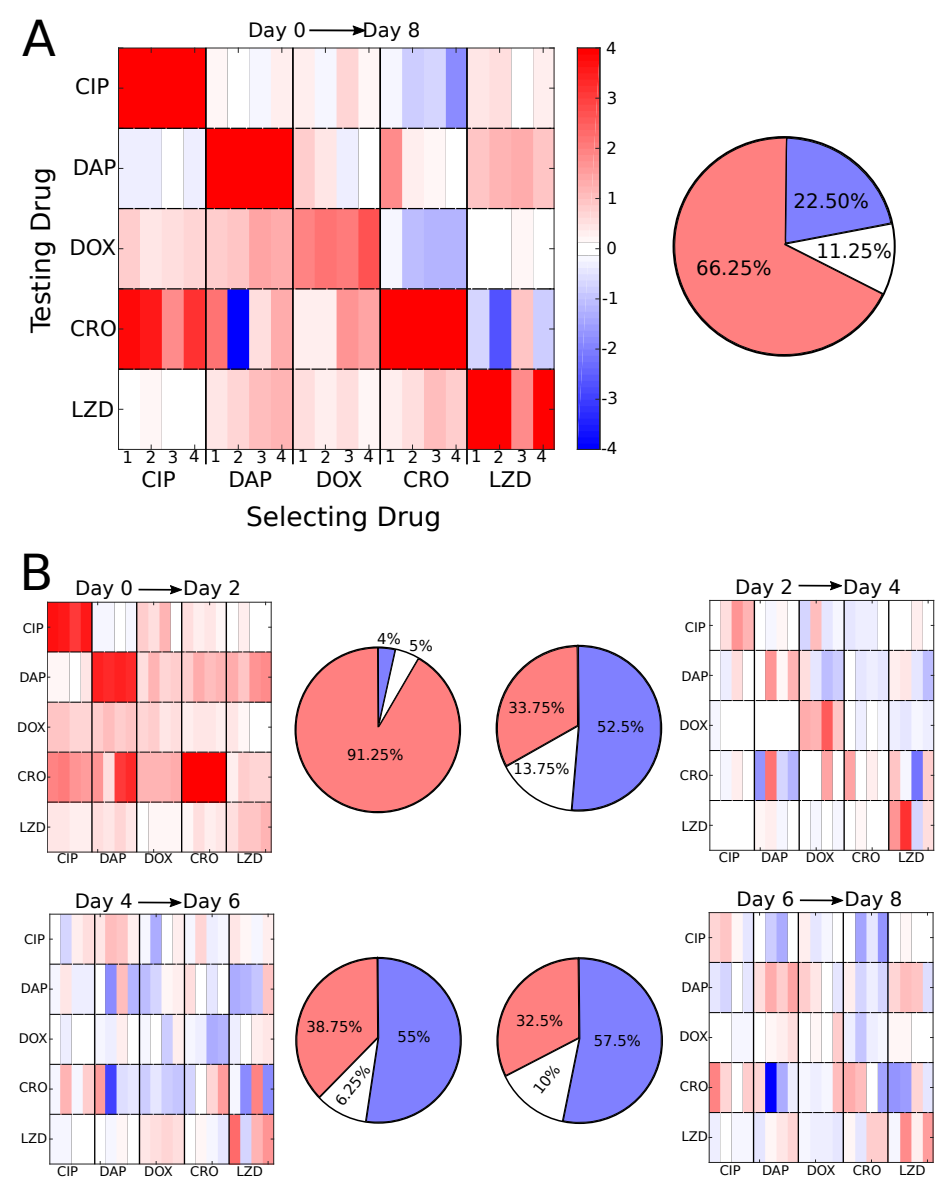

Fig 2. Early evolution is dominated by collateral resistance followed by a diverse set of collateral responses as evolution continues. (A) Left: Collateral profiles measured at the final time point (day 8), relative to the day 0 ancestor. Resistance (red) or sensitivity (blue) to each antibiotic is quantified via the $\log _{2}$-transformed fold change in $\mathrm{IC}_{50}$ relative to the ancestral strain (V583). A measurement is deemed to be collaterally resistant or sensitive if it falls above or below 3 times the standard error of the mean of the wild-type $\left(|c|>3 \sigma_{W T}\right)$. Right: Pie chart representing the fraction of collateral effects that confer resistance (red), sensitivity (blue) or no statistical change (white/gray). (B) Collateral profiles measured for the same 20 mutants as A, however each heatmap represents the collateral effects that occurred over the previous two days of evolution (days 0-2, top left; days 2-4, top right; days 4-6, bottom left; days 6-8, bottom right). Over 90 percent of collateral effects conferred between days $0-2$ resulted in collateral resistance. The subsequent 6 days of evolution (days $2-4,4-6$, and 6-8) only conferred collateral resistance $34 \%, 39 \%$ and $33 \%$ respectively. 
model's simplicity, we can exhaustively simulate all possible trajectories in hopes of identifying candidate drugs for further experiments.

Using this model, we simulated 8-day trajectories using every possible combination of 5 drugs, with drugs potentially alternated every 2 days (days $0,2,4$, and 6 ). Using these results, we calculated the "applied drug resistance", which we define as a cumulative measure of the collateral effects for the applied drugs across all time-steps. It is given by $R_{\text {app }}=\sum_{n} S_{j_{n}}(n)$, where the sum runs over all time steps of the treatment and $S_{j_{n}}(n)$ is the component of the state vector corresponding to the applied $\operatorname{drug} j_{n}$ at each step (Fig $3 \mathrm{~A}$ ). Large positive values of $R_{\text {app }}$ correspond to treatments with, on average, high levels of resistance to the applied drugs, while large negative values correspond to high levels of sensitivity.

We find that the applied drug resistance $R_{\text {app }}$ varies substantially depending on both a) the number of different drugs in the sequence and b) the specific, time-dependent ordering of those drugs (Fig. 3A). As the number of drugs used in the policy increases from 2 to 4 (Fig. 3A top to bottom, black curves), the distribution of $R_{\text {app }}$ narrows and the mean decreases. Intuitively, the decreasing mean is perhaps not surprising, as switching between multiple drugs is expected to create an increasingly difficult evolutionary challenge. Surprisingly, however, at a global level both the best (smallest $R_{\text {app }}$ ) and worst (largest $R_{\text {app }}$ ) schedules require switching between multiple drugs, indicating that drug are not always an improvement over single (constant) drug schedules (red curves).

\section{Switching drugs as a tax that raises global resistance to the set of available drugs.}

We next used simulation data to calculate the "total resistance", which we define as the cumulative sum of all entries of the state vector over the treatment period,

$R_{\mathrm{tot}}=\sum_{n} \sum_{j} S_{j}(n)$, where $S_{j}(n)$ is the $j$-th entry of the state vector at time point $n$. This quantity is a global measure of resistance or sensitivity to all available drugs. In contrast to $R_{\mathrm{app}}$, the schedules that lead to the lowest levels of $R_{\text {tot }}$ (total resistance) all correspond to single-drug schedules (Fig. 3B). Despite the fact that single drug schedules are unable to exploit collateral sensitivities that may arise, they are more effective at minimizing global resistance to the pool of drugs, in part because early stages of adaptation are dominated by costly collateral resistance. That is, the fact that collateral resistance occurs frequently when populations are exposed to a new drug (see Fig. 2) acts as an effective "tax" on switching drugs. Each time a new drug is used for the first time, it is overwhelmingly likely that you add resistance not just to that drug, but to many of the other available drugs.

\section{Drug timing frequently impacts applied drug resistance but not total resistance}

To evaluate the impact of the timing and number of drug switches in a treatment protocol, we compared the best (smallest value of $R_{\text {app }}$ or $R_{\text {tot }}$ ) and worst (largest value of $R_{\text {app }}$ or $R_{\mathrm{tot}}$ ) treatment outcomes for an exhaustive combination of every 2-drug treatment in our study (Fig $3 \mathrm{C}-\mathrm{D})$. We found that even when restricted to policies involving only 2 drugs, the best schedule can dramatically outperform the worst schedule in terms of applied drug resistance, suggesting that the timing of the switches, not merely the drugs used, can have a significant impact. For example, for the drug pair DOX-CRO, using the pair optimally leads to a small applied drug resistance score $\left(R_{\text {app }} \approx 3.6\right)$, whereas using the drug pair sub-optimally leads to a significantly larger score $\left(R_{\mathrm{app}} \approx 14\right)$. On the other hand, when it comes to the total collateral profile 
bioRxiv preprint doi: https://doi.org/10.1101/2021.12.19.473361; this version posted December 21,2021 . The copyright holder for this preprint (which was not certified by peer review) is the author/funder, who has granted bioRxiv a license to display the preprint in perpetuity. It is made available under aCC-BY-NC-ND 4.0 International license.

\section{A Applied drug resistance}
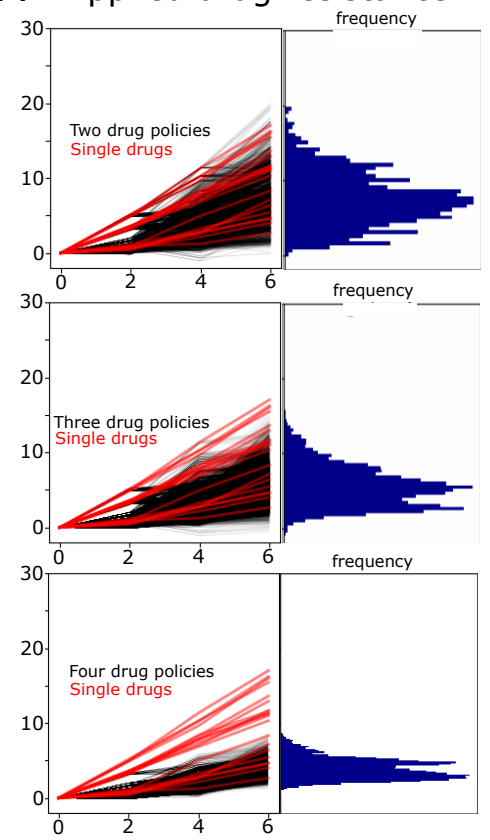

C Applied drug resistance

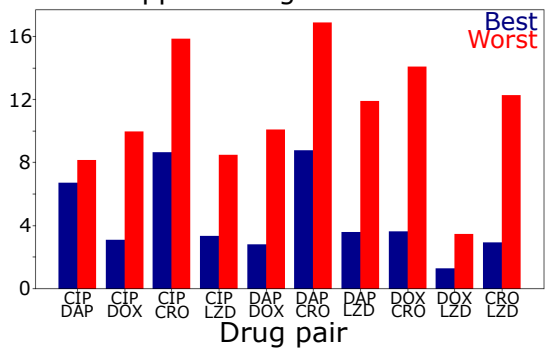

B Total resistance to drugs
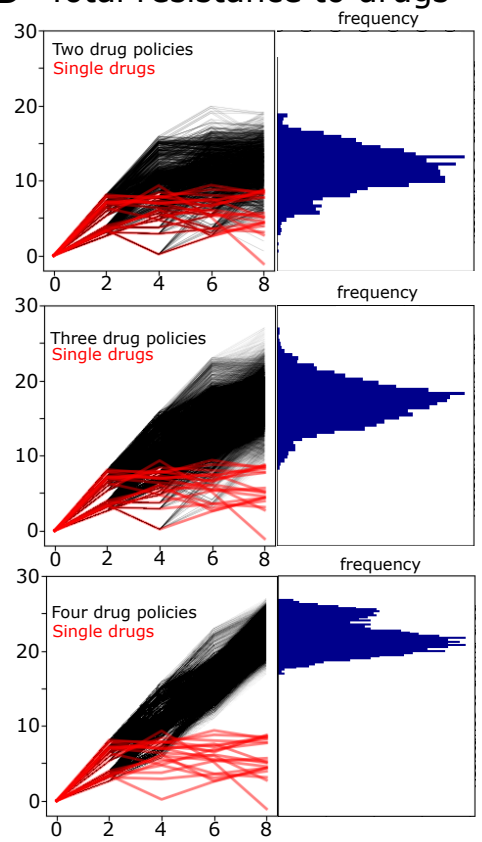

D

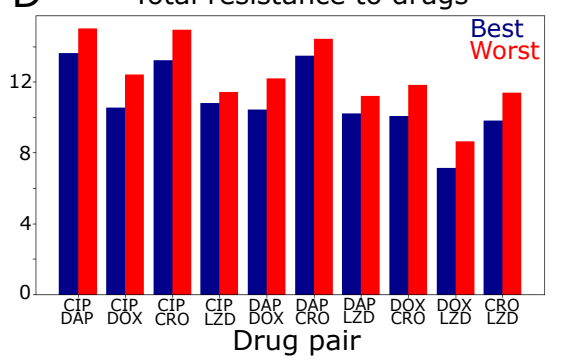

Fig 3. Simulations suggest optimal dosing can be aided or hindered by temporally resolved collateral profiles. (A) Given our temporally resolved collateral profiles measured above, we exhaustively simulate every hypothetical trajectory assuming any of the five drugs can be chosen at days $0,2,4$ and 6 . It is assumed that if it is the first time the population is experiencing a drug, any of the four measured replicates are accessible to the population. However, once a population has chosen a trajectory in an antibiotic, further use of that antibiotic will stay on that chosen trajectory. For example, a dose schedule of CIP-CIP-CIP-CIP would result in selecting one of the four CIP replicates measured in the heatmap corresponding to days 0-2 evolution (e.g. CIP2), followed by that replicates measured results for days 2-4, 4-6 and 6-8. A running total of the resistance of the population to the applied drug is plotted both for the trajectories where more than one drug is chosen (black) and for the single drug trajectories (red). The top box plots all two drug policies, the middle box plots all three drug policies and the final box plots all four drug policies. (B) The same calculation is performed, but we instead plot the sum of the collateral profile to each antibiotic, as opposed to just the currently applied antibiotic. (C) The best and worst performing drug-cycling policy for each drug pair is shown using the running total of resistance to the applied drug (as in A). (D) The best and worst performing drug-cycling policy for each drug pair is shown using the sum collateral profile as the metric (as in B). 
resistance (Fig 3D), the timing of the drug switches has a relatively small impact for all drug pairs.

209

\section{Success of switching to a second antibiotic is contingent on temporally dynamic collateral effects.}

Our results indicate that the dynamic nature of collateral sensitivity may jeopardize otherwise effective drug sequences. To investigate this issue experimentally, we designed an evolution experiment (Fig $4 \mathrm{~A}$ ) meant to approximate a hypothetical drug-switching protocol involving two drugs (CRO and DOX) suggested by simulations to be particularly sensitive to the timing of drug switching (i.e. large difference between best and worst treatments, Fig $3 \mathrm{C}$ ). Similar to our previous experiments, we performed serial passage evolution using escalating concentrations of the antibiotic CRO but now for a total period of 14 days. At the end of each day, we exposed a diluted sample of that population to varying concentrations of a second antibiotic, DOX, to probe how switching to a second drug may have increased or decreased the treatment efficacy. The entire process was repeated for 20 independent populations to quantify evolutionary variability (Fig 4B).
211

212

213

214

215

216

217

218

219

220

221

222

223

224 
A

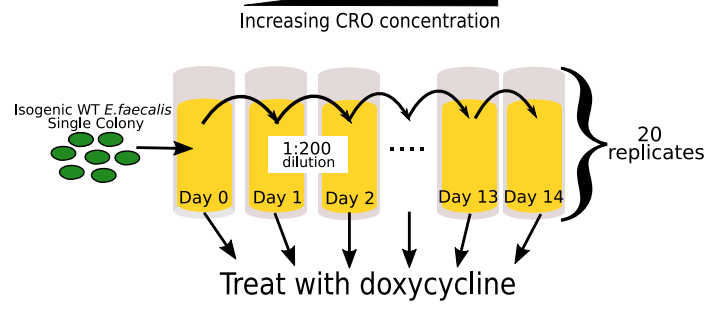

B
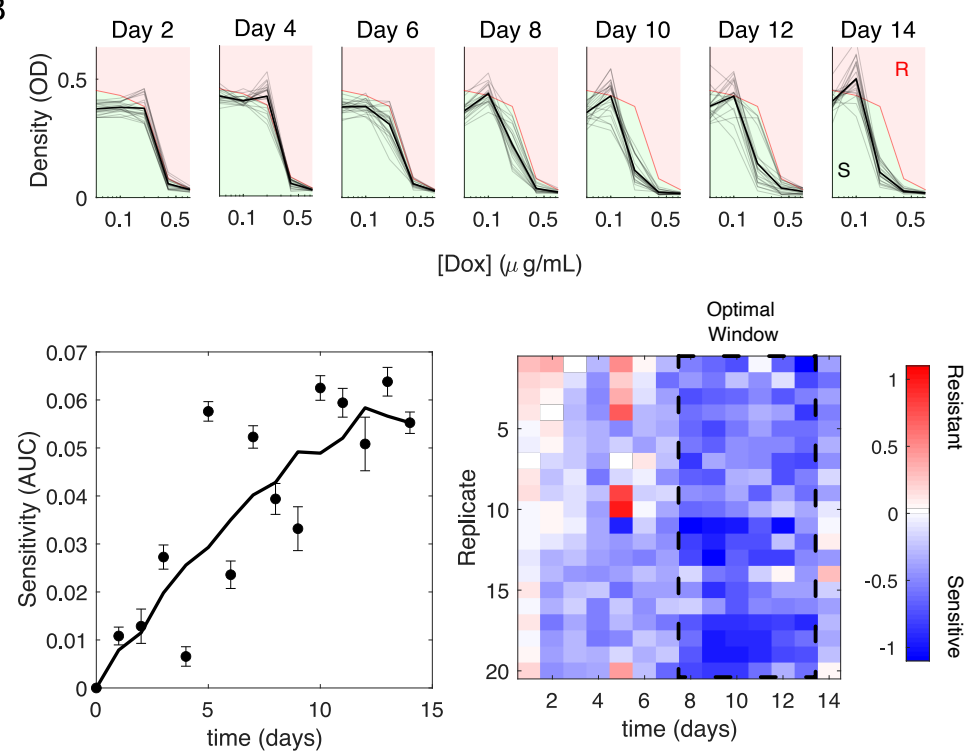

Fig 4. Success of switching to a second antibiotic is contingent on temporally dynamic collateral effects. (A) Twenty replicate populations of E. faecalis V583 were exposed to increasing concentrations of the antibiotic ceftrixone (CRO) over the course of 14 days via serial passage. At the end of each day a sample of the population was isolated and cultured in a range of doxycycline (DOX) concentrations for 24 hours. (B) Top: Density (OD) after 24 hours vs drug (DOX) concentration for all 20 populations (light gray), and the mean over the 20 populations (dark black) at different days of the evolution experiment. For comparison, the dose response curve in the ancestral strain (red curve) divides the space into regions of increased resistance (red) and increased sensitivity (green). (B) Bottom left: Average sensitivity over 20 populations (error bar standard error of the mean) at different time points. Sensitivity is defined as the difference in area under the curve between the ancestral dose response curve and the dose response curve of the population in question between the lowest $([\mathrm{Dox}]=0.05 \mathrm{\mu g} / \mathrm{mL})$ and highest $([\mathrm{Dox}]=0.8 \mathrm{\mu g} / \mathrm{mL})$ nonzero drug concentrations. Solid line is a moving average. (B) Bottom right: Collateral effects (quantified, as before, by $\log _{2}$-transformed fold change in the $\mathrm{IC}_{50}$ ). Dashed region highlights a transient six-day window where collateral sensitivity is more pronounced. 
To quantify sensitivity to DOX, we calculated both a global area-under-the-curve sensitivity score, where we take the difference between the area under the dose response curve of the ancestral strain and each evolved population (Fig 4B, bottom left), as well as the previously described collateral effect metric (i.e. the log-scaled fold change in half-maximal inhibitory concentration). Both metrics show that populations adapted to CRO become increasingly sensitized to DOX over time. The sensitivity is particularly notable between days 8 and 13, where collateral sensitivity is maximized before starting to decline (Fig 4B, bottom right). Switching to DOX at time points before or after that window produces low levels of average sensitivty and even leads to resistance in some populations. These results indicate that the effects of a new antibiotic can vary considerably depending on when, along the adaptation trajectory, the new drug is applied.

\section{Discussion}

Our work provides systematic evidence of temporal collateral drug effects in the pathogen E. faecalis. We show that temporal effects are pervasive and heterogeneous across several clinically relevant drug classes. Our results reveal that early (2 days or less) stage adaptation produces almost exclusively collateral resistance, while further evolution results in diverse collateral effects. In addition, collateral effects at one time point are not obviously related with effects at later time points, suggesting that the effects are correlated only on time periods smaller than our measurement time (2 days, or approximately 10-20 generations). Combinatorial simulations with a simple model suggest that switching drugs can lead to both better and worse outcomes than single drug treatments, a consequence of the specific timing and frequency of drug switching. In particular, our data suggests that switching to a new drug is often associated with an increased propensity to generate collateral resistance, creating an effective a "drug switching tax" that renders single-drug schedules optimal for minimizing global resistance to a set of available drugs. Finally, we showed experimentally that time-dependent collateral effects can create optimal dosing windows where sensitivity is maximized. Taken together, these results highlight the potential importance dynamics in the evolution of collateral profiles. The time-dependent nature of collateral sensitivity and collateral resistance presents both additional challenges and new opportunities for designing multi-drug therapies to slow resistance. Full optimization of sequential drug therapies will likely involve not merely static, end-point measurements of collateral effects, but a full description of their temporal development.

The goal of this study was to broadly survey phenotypic resistance patterns over time in a systematic way. This approach comes with obvious drawbacks, and we are left with many unanswered questions. Most notably, our work does not provide any information about the molecular mechanisms underlying the collateral effects; such insight, while hard won even for a single pair of drugs, will be essential to fully exploit the phenotypic effects observed here. In addition, we focused on a single bacterial species, and all experiments were performed starting from the ancestral V583 E. faecalis strain. It is not clear how the results might change in a different strain or species. Indeed, recent work underscores just how important genotype can be in antibiotic evolvability [71], and the search for more general patterns is ongoing 35, 51, 53. Finally, we used an extremely simple additive model of resistance to simulate possible evolutionary trajectories. While additive models can be useful to guiding experiments, particularly on short timescales [40,44], and have shown to be surprisingly robust in some contexts 72], we expect them to ultimately fail when epistasis or evolutionary hystersis are strong 73 . We therefore reiterate that the primary purpose of such simple models is to generate hypotheses to guide experiments, not to provide a true theoretical 
description of the system.

Finally, it is important to keep in mind the scope of our work. These evolution experiments are done in a highly controlled laboratory environment. Our protocols are not meant to guide clinicians, but instead focus on whether or not collateral profiles changed even in the simplest of drug-evolution environments - free, for example, of the important but difficult-to-quantify interactions between host and pathogen. Translating these results into a clinically accurate model would require additional work to understand the mechanistic, clinical, and even theoretical principles governing drug sequence optimization. This work serves as a reminder of the complexities of evolution and the still long path we must walk to confidently prescribe effective dosing schedules in patients. At the same time, the results highlight the rich dynamical behavior of collateral sensitivity in even simplified laboratory populations, offering a largely unexplored frontier for evolution-based control strategies.

\section{Materials and methods}

\section{Strains, antibiotics, and media}

All resistance evolution lineages were derived from an E faecalis V583 ancestor, a fully sequenced clinical isolate with vancomycin resistance 74 . The 5 antibiotics used in this study and their mechanisms of action are listed in Table 1 . Antibiotics were prepared from powder stock and stored at appropriate temperature. Evolution and $\mathrm{IC}_{50}$ measurements were conducted in BHI (brain heart infusion).

\section{Laboratory evolution experiments}

Evolution experiments were performed in replicates of four. Daily serial passage evolutions were conducted in $1 \mathrm{~mL}$ BHI medium in 96-well plates with a maximum volume of $2 \mathrm{~mL}$. Each day populations were grown in three antibiotic concentrations spanning sub- and super-MIC doses. After approximately 16 hours of incubation at $37^{\circ} \mathrm{C}$, the well with the highest drug concentration that contained visible growth was propagated into three new concentrations (typically one-half, $2 \mathrm{x}$ and $4 \mathrm{x}$ the highest concentration that had visible growth). A 1/200 dilution was used as an inoculum for the next day's evolution plate. This process was repeated for 8 days for the multi-drug study and 14 days for the CRO/DOX study. All strains were stocked in 30\% glycerol and subsequently plated on pure BHI plates for further experimentation. A single colony was selected for $\mathrm{IC}_{50}$ determination. To help ensure no contamination occured, cells were regularly plated and visualized using DIC microscopy to ensure E. faecalis morphology.

\section{Measuring drug resistance and sensitivity}

$\mathrm{IC}_{50}$ determination experiments were performed in 96-well plates by exposing each strain to a drug gradient consisting of between 6-14 concentrations, typically in linear dilution series prepared in BHI medium with a total volume of $205 \mu \mathrm{L}(200 \mu \mathrm{L} \mathrm{BHI}$, $5 \mu \mathrm{L}$ of $1.5 \mathrm{OD}$ cells) in each well. After 20 hours, we measured the OD at $600 \mathrm{~nm}$ via an Enspire Multi-modal Plate Reader (Perkin Elmer) with an automated plate stacker. OD measurements for each drug were normalized by the OD600 in the absence of drug.

In order to quantify resistance to each drug, the OD600-generated dose-response curve was fit to a Hill-like function $f(x)=\left(1+\left(\frac{x}{K}\right)^{h}\right)^{-1}$ using a nonlinear least squares fitting. $K$ is the $\mathrm{IC}_{50}$ and $h$ is a Hill coefficient that represents the steepness of the dose-response curve. Strains were deemed "collaterally resistant" or "collaterally 
sensitive" if its $\mathrm{IC}_{50}$ had increased or decreased by more than 3 times the standard error of the wild-type mean $\mathrm{IC}_{50}$.

320 321

\section{References}

1. Boucher HW, Talbot GH, Bradley JS, Edwards JE, Gilbert D, Rice LB, et al. Bad bugs, no drugs: no ESKAPE! An update from the Infectious Diseases Society of America. Clinical infectious diseases. 2009;48(1):1-12.

2. Goldberg DE, Siliciano RF, Jacobs Jr WR. Outwitting evolution: fighting drug-resistant TB, malaria, and HIV. Cell. 2012;148(6):1271-1283.

3. Pfaller MA. Antifungal drug resistance: mechanisms, epidemiology, and consequences for treatment. The American journal of medicine. 2012;125(1):S3-S13.

4. Raviglione M, Marais B, Floyd K, Lönnroth K, Getahun H, Migliori GB, et al. Scaling up interventions to achieve global tuberculosis control: progress and new developments. The Lancet. 2012;379(9829):1902-1913.

5. Borst P. Cancer drug pan-resistance: pumps, cancer stem cells, quiescence, epithelial to mesenchymal transition, blocked cell death pathways, persisters or what? Open biology. 2012;2(5):120066.

6. Pluchino KM, Hall MD, Goldsborough AS, Callaghan R, Gottesman MM. Collateral sensitivity as a strategy against cancer multidrug resistance. Drug Resistance Updates. 2012;15(1-2):98-105.

7. Brown EM, Nathwani D. Antibiotic cycling or rotation: a systematic review of the evidence of efficacy. Journal of Antimicrobial Chemotherapy. 2005;55(1):6-9. doi:10.1093/jac/dkh482.

8. Bergstrom CT, Lo M, Lipsitch M. Ecological theory suggests that antimicrobial cycling will not reduce antimicrobial resistance in hospitals. Proceedings of the National Academy of Sciences. 2004;101(36):13285-13290. doi:10.1073/pnas.0402298101.

9. Nichol D, Jeavons P, Fletcher AG, Bonomo RA, Maini PK, Paul JL, et al. Steering evolution with sequential therapy to prevent the emergence of bacterial antibiotic resistance. PLoS Comput Biol. 2015;11(9):e1004493.

10. Batra A, Roemhild R, Rousseau E, Franzenburg S, Niemann S, Schulenburg H. High potency of sequential therapy with only $\beta$-lactam antibiotics. Elife. 2021;10:e68876.

11. Zhang Q, Lambert G, Liao D, Kim H, Robin K, Tung Ck, et al. Acceleration of emergence of bacterial antibiotic resistance in connected microenvironments. Science. 2011;333(6050):1764-1767.

12. Baym M, Lieberman TD, Kelsic ED, Chait R, Gross R, Yelin I, et al. Spatiotemporal microbial evolution on antibiotic landscapes. Science. 2016;353(6304):1147-1151.

13. De Jong MG, Wood KB. Tuning spatial profiles of selection pressure to modulate the evolution of drug resistance. Physical review letters. 2018;120(23):238102. 
14. Feder AF, Harper K, Brumme CJ, Pennings PS. Understanding patterns of HIV multi-drug resistance through models of temporal and spatial drug heterogeneity. bioRxiv. 2021; p. 807560.

15. Yurtsev EA, Chao HX, Datta MS, Artemova T, Gore J. Bacterial cheating drives the population dynamics of cooperative antibiotic resistance plasmids. Molecular systems biology. 2013;9(1):683.

16. Meredith HR, Lopatkin AJ, Anderson DJ, You L. Bacterial temporal dynamics enable optimal design of antibiotic treatment. PLoS Comput Biol. 2015;11(4):e1004201.

17. Meredith HR, Srimani JK, Lee AJ, Lopatkin AJ, You L. Collective antibiotic tolerance: mechanisms, dynamics and intervention. Nature chemical biology. 2015;11(3):182.

18. Farrokhian N, Maltas J, Dinh M, Durmaz A, Ellsworth P, Hitomi M, et al. Measuring competitive exclusion in non-small cell lung cancer. bioRxiv. 2021;doi:10.1101/2020.09.18.303966.

19. Gatenby RA, Silva AS, Gillies RJ, Frieden BR. Adaptive therapy. Cancer research. 2009;69(11):4894-4903.

20. West J, You L, Zhang J, Gatenby RA, Brown JS, Newton PK, et al. Towards multidrug adaptive therapy. Cancer research. 2020;80(7):1578-1589.

21. Hansen E, Karslake J, Woods RJ, Read AF, Wood KB. Antibiotics can be used to contain drug-resistant bacteria by maintaining sufficiently large sensitive populations. PLoS biology. 2020;18(5):e3000713.

22. Zimmer A, Katzir I, Dekel E, Mayo AE, Alon U. Prediction of multidimensional drug dose responses based on measurements of drug pairs. Proceedings of the National Academy of Sciences. 2016;113(37):10442-10447.

23. Wood K, Nishida S, Sontag ED, Cluzel P. Mechanism-independent method for predicting response to multidrug combinations in bacteria. Proceedings of the National Academy of Sciences. 2012;109(30):12254-12259.

24. Torella JP, Chait R, Kishony R. Optimal drug synergy in antimicrobial treatments. PLoS Comput Biol. 2010;6(6):e1000796.

25. Michel JB, Yeh PJ, Chait R, Moellering RC, Kishony R. Drug interactions modulate the potential for evolution of resistance. Proceedings of the National Academy of Sciences. 2008;105(39):14918-14923.

26. Dean Z, Maltas J, Wood K. Antibiotic interactions shape short-term evolution of resistance in E. faecalis. PLoS pathogens. 2020;16(3):e1008278.

27. Jahn LJ, Simon D, Jensen M, Bradshaw C, Ellabaan MMH, Sommer MOA. Compatibility of evolutionary responses to constituent antibiotics drive resistance evolution to drug pairs. Molecular Biology and Evolution. 2021;.

28. Tekin E, Beppler C, White C, Mao Z, Savage VM, Yeh PJ. Enhanced identification of synergistic and antagonistic emergent interactions among three or more drugs. Journal of The Royal Society Interface. 2016;13(119):20160332.

29. Gjini E, Wood KB. Price equation captures the role of drug interactions and collateral effects in the evolution of multidrug resistance. Elife. 2021;10:e64851. 
30. Barbosa C, Römhild R, Rosenstiel P, Schulenburg H. Evolutionary stability of collateral sensitivity to antibiotics in the model pathogen Pseudomonas aeruginosa. Elife. 2019;8:e51481.

31. Maltas J, McNally DM, Wood KB. Evolution in alternating environments with tunable interlandscape correlations. Evolution. 2021;75(1):10-24.

32. Lázár V, Pal Singh G, Spohn R, Nagy I, Horváth B, Hrtyan M, et al. Bacterial evolution of antibiotic hypersensitivity. Molecular systems biology. 2013;9(1):700.

33. Lázár V, Nagy I, Spohn R, Csörgő B, Györkei Á, Nyerges Á, et al. Genome-wide analysis captures the determinants of the antibiotic cross-resistance interaction network. Nature communications. 2014;5(1):1-12.

34. Imamovic L, Sommer MO. Use of collateral sensitivity networks to design drug cycling protocols that avoid resistance development. Science translational medicine. 2013;5(204):204ra132-204ra132.

35. Imamovic L, Ellabaan MMH, Machado AMD, Citterio L, Wulff T, Molin S, et al. Drug-driven phenotypic convergence supports rational treatment strategies of chronic infections. Cell. 2018;172(1-2):121-134.

36. Roemhild R, Linkevicius M, Andersson DI. Molecular mechanisms of collateral sensitivity to the antibiotic nitrofurantoin. PLoS biology. 2020;18(1):e3000612.

37. Zhao B, Sedlak JC, Srinivas R, Creixell P, Pritchard JR, Tidor B, et al. Exploiting temporal collateral sensitivity in tumor clonal evolution. Cell. 2016;165(1):234-246.

38. Ardell SM, Kryazhimskiy S. The population genetics of collateral resistance and sensitivity. eLife. 2021;10:e73250.

39. Herencias C, Rodríguez-Beltrán J, León-Sampedro R, Alonso-del Valle A, Palkovičová J, Cantón R, et al. Collateral sensitivity associated with antibiotic resistance plasmids. Elife. 2021;10:e65130.

40. Maltas J, Wood KB. Pervasive and diverse collateral sensitivity profiles inform optimal strategies to limit antibiotic resistance. PLoS biology.

2019;17(10):e3000515.

41. Barbosa C, Trebosc V, Kemmer C, Rosenstiel P, Beardmore R, Schulenburg H, et al. Alternative evolutionary paths to bacterial antibiotic resistance cause distinct collateral effects. Molecular biology and evolution. 2017;34(9):2229-2244.

42. Oz T, Guvenek A, Yildiz S, Karaboga E, Tamer YT, Mumcuyan N, et al. Strength of selection pressure is an important parameter contributing to the complexity of antibiotic resistance evolution. Molecular biology and evolution. 2014;31(9):2387-2401.

43. Nichol D, Rutter J, Bryant C, Hujer AM, Lek S, Adams MD, et al. Antibiotic collateral sensitivity is contingent on the repeatability of evolution. Nature communications. 2019;10(1):1-10.

44. Maltas J, Krasnick B, Wood KB. Using selection by nonantibiotic stressors to sensitize bacteria to antibiotics. Molecular biology and evolution.

2020;37(5):1394-1406. 
45. Bhardwaj P, Hans A, Ruikar K, Guan Z, Palmer KL. Reduced chlorhexidine and daptomycin susceptibility in vancomycin-resistant Enterococcus faecium after serial chlorhexidine exposure. Antimicrobial agents and chemotherapy. 2018;62(1).

46. Lázár V, Martins A, Spohn R, Daruka L, Grézal G, Fekete G, et al. Antibiotic-resistant bacteria show widespread collateral sensitivity to antimicrobial peptides. Nature microbiology. 2018;3(6):718-731.

47. Knöppel A, Näsvall J, Andersson DI. Evolution of antibiotic resistance without antibiotic exposure. Antimicrobial agents and chemotherapy. 2017;61(11).

48. Creamer KE, Ditmars FS, Basting PJ, Kunka KS, Hamdallah IN, Bush SP, et al. Benzoate-and salicylate-tolerant strains of Escherichia coli K-12 lose antibiotic resistance during laboratory evolution. Applied and environmental microbiology. 2017;83(2):e02736-16.

49. Moore JP, Li H, Engmann ML, Bischof KM, Kunka KS, Harris ME, et al. Inverted regulation of multidrug efflux pumps, acid resistance, and porins in benzoate-evolved Escherichia coli K-12. Applied and environmental microbiology. 2019;85(16):e00966-19.

50. Iram S, Dolson E, Chiel J, Pelesko J, Krishnan N, Güngör Ö, et al. Controlling the speed and trajectory of evolution with counterdiabatic driving. Nature Physics. 2020; p. 1-8.

51. Roemhild R, Andersson DI. Mechanisms and therapeutic potential of collateral sensitivity to antibiotics. PLoS Pathogens. 2021;17(1):e1009172.

52. de Evgrafov MCR, Faza M, Asimakopoulos K, Sommer MO. Systematic investigation of resistance evolution to common antibiotics reveals conserved collateral responses across common human pathogens. Antimicrobial Agents and Chemotherapy. 2020;65(1).

53. Beckley AM, Wright ES. Identification of antibiotic pairs that evade concurrent resistance via a retrospective analysis of antimicrobial susceptibility test results. The Lancet Microbe. 2021;2(10):e545-e554.

54. Dhawan A, Nichol D, Kinose F, Abazeed ME, Marusyk A, Haura EB, et al. Collateral sensitivity networks reveal evolutionary instability and novel treatment strategies in ALK mutated non-small cell lung cancer. Scientific reports. 2017;7(1):1-9.

55. Gilmore MS, Clewell DB, Ike Y, Shankar N. Enterococci: From Commensals to Leading Causes of Drug Resistant Infection [Internet]. 2014;.

56. O'Driscoll T, Crank CW. Vancomycin-resistant enterococcal infections: epidemiology, clinical manifestations, and optimal management. Infection and drug resistance. 2015;8:217.

57. Cetinkaya Y, Falk P, Mayhall CG. Vancomycin-resistant enterococci. Clinical microbiology reviews. 2000;13(4):686-707.

58. Huycke MM, Sahm DF, Gilmore MS. Multiple-drug resistant enterococci: the nature of the problem and an agenda for the future. Emerging infectious diseases. $1998 ; 4(2): 239$. 
59. Palmer KL, Daniel A, Hardy C, Silverman J, Gilmore MS. Genetic basis for daptomycin resistance in enterococci. Antimicrobial agents and chemotherapy. 2011;55(7):3345-3356.

60. Miller C, Kong J, Tran TT, Arias CA, Saxer G, Shamoo Y. Adaptation of Enterococcus faecalis to daptomycin reveals an ordered progression to resistance. Antimicrobial agents and chemotherapy. 2013;57(11):5373-5383.

61. Munoz-Price LS, Lolans K, Quinn JP. Emergence of resistance to daptomycin during treatment of vancomycin-resistant Enterococcus faecalis infection. Clinical infectious diseases. 2005;41(4):565-566.

62. Balli EP, Venetis CA, Miyakis S. Systematic review and meta-analysis of linezolid versus daptomycin for treatment of vancomycin-resistant enterococcal bacteremia. Antimicrobial agents and chemotherapy. 2014;58(2):734-739.

63. Auckland C, Teare L, Cooke F, Kaufmann ME, Warner M, Jones G, et al. Linezolid-resistant enterococci: report of the first isolates in the United Kingdom. Journal of Antimicrobial Chemotherapy. 2002;50(5):743-746.

64. Kelesidis T, Humphries R, Uslan DZ, Pegues DA. Daptomycin nonsusceptible enterococci: an emerging challenge for clinicians. Clinical Infectious Diseases. 2011;52(2):228-234.

65. Du D, Wang-Kan X, Neuberger A, van Veen HW, Pos KM, Piddock LJ, et al. Multidrug efflux pumps: structure, function and regulation. Nature Reviews Microbiology. 2018;16(9):523-539.

66. Jonas BM, Murray BE, Weinstock GM. Characterization of emeA, anorA Homolog and Multidrug Resistance Efflux Pump, in Enterococcus faecalis. Antimicrobial Agents and Chemotherapy. 2001;45(12):3574-3579.

67. Lee EW, Huda MN, Kuroda T, Mizushima T, Tsuchiya T. EfrAB, an ABC multidrug efflux pump in Enterococcus faecalis. Antimicrobial agents and chemotherapy. 2003;47(12):3733-3738.

68. Duval V, Lister IM. MarA, SoxS and Rob of Escherichia coli-Global regulators of multidrug resistance, virulence and stress response. International journal of biotechnology for wellness industries. 2013;2(3):101.

69. Sukhai M, Piquette-Miller M. Regulation of the multidrug resistance genes by stress signals. J Pharm Pharm Sci. 2000;3(2):268-280.

70. Cordell HJ. Epistasis: what it means, what it doesn't mean, and statistical methods to detect it in humans. Human molecular genetics. 2002;11(20):2463-2468.

71. Card KJ, LaBar T, Gomez JB, Lenski RE. Historical contingency in the evolution of antibiotic resistance after decades of relaxed selection. PLoS biology. 2019;17(10):e3000397.

72. Russ D, Kishony R. Additivity of inhibitory effects in multidrug combinations. Nature microbiology. 2018;3(12):1339-1345.

73. Roemhild R, Gokhale CS, Dirksen P, Blake C, Rosenstiel P, Traulsen A, et al. Cellular hysteresis as a principle to maximize the efficacy of antibiotic therapy. Proceedings of the National Academy of Sciences. 2018;115(39):9767-9772. 
bioRxiv preprint doi: https://doi.org/10.1101/2021.12 19.473361; this version posted December 21, 2021. The copyright holder for this preprint (which was not certified by peer review) is the author/funder, who has granted bioRxiv a license to display the preprint in perpetuity. It is made available under aCC-BY-NC-ND 4.0 International license.

74. Sahm DF, Kissinger J, Gilmore MS, Murray PR, Mulder R, Solliday J, et al. In vitro susceptibility studies of vancomycin-resistant Enterococcus faecalis. Antimicrobial agents and chemotherapy. 1989;33(9):1588-1591. 


\section{Supplemental Material: Temporally dynamic collateral sensitivity profiles complicate optimal dosing strategies}
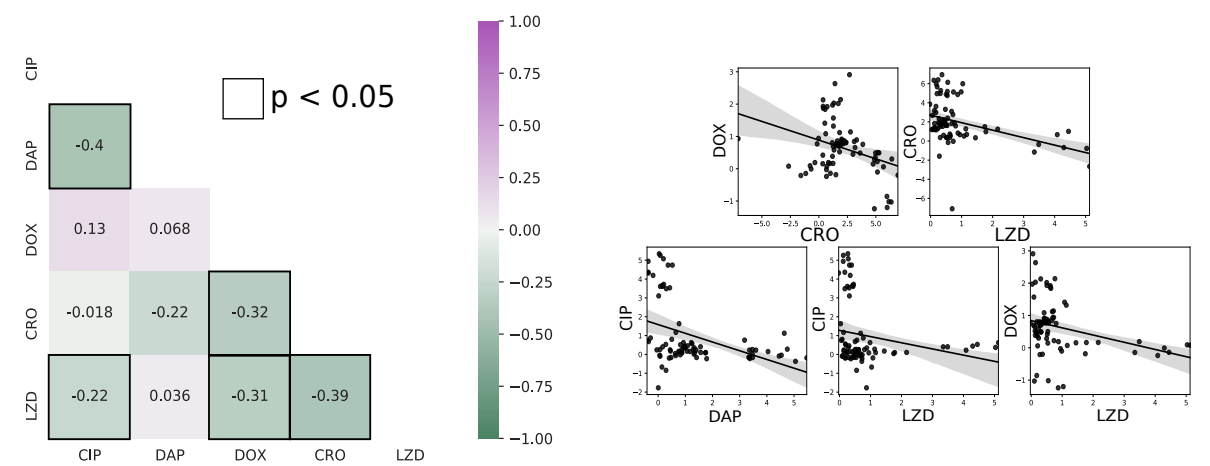

Fig S1. Correlation between collateral effects between different testing antibiotics. Left panel: Pearson correlation coefficient between collateral profiles between each of the 5 testing antibiotics. Dark squares highlight correlations with statistically significant correlations $(P<0.05)$, whose distributions are shown as scatter plots. Right panel: pairwise scatter plots of resistance profiles selected by different testing antibiotics; only pairs with significant correlations $(P<0.05)$ are shown. Each point is the measured resistance to each of the single antibiotics labeled on the axes. 